\title{
Electronic structure and mechanical stability of the graphitic honeycomb lattice
}

\author{
Noejung Park and Jisoon Ihm \\ Department of Physics, Seoul National University, Seoul 151-742, Korea
}

(Received 3 March 2000; revised manuscript received 16 May 2000)

\begin{abstract}
A family of crystal structures of carbon composed of alternating $s p^{2}$ and $s p^{3}$ bonds is investigated. Graphitic strips are connected by $s p^{3}$ bonds to form an array of hexagonal pillars exhibiting a honeycomb lattice in the perpendicular plane. The electronic structure and elastic properties of this family of structures are calculated using an ab initio pseudopotential as well as the environment-dependent tight-binding method. Their electronic structure has a similar size dependence to zigzag nanotubes; they are metallic if twice the strip width is a multiple of three hexagonal units, and otherwise semiconducting with a wider range of the band gap than for carbon nanotubes. The structural stability is studied and compared with other carbon structures.
\end{abstract}

\section{INTRODUCTION}

Carbon forms stable crystals of $s p^{3}$ or $s p^{2}$ hybrid orbitals under ambient conditions. Among the crystals of pure $s p^{3}$ bonding are cubic diamond, hexagonal diamond, and the fourfold-coordinated metastable BC- 8 structure. ${ }^{1}$ On the other hand, there exist crystals of $s p^{2}$ bonding such as various phases of graphite and carbon nanotubes, which have attracted much interest recently due to their unusual onedimensional structure. In graphite and carbon nanotubes, the $s p^{2}$ bonding gives rise to a threefold-coordinated planar structure and the remaining $p_{z}$ orbitals perpendicular to the plane produce interesting electronic structures near the Fermi level. In addition to pure $s p^{2}$ or $s p^{3}$ crystals, there has been research on mixed structures of $s p^{2}$ and $s p^{3}$ bondings., ${ }^{2,3}$ Here we focus our attention on the structures suggested by Karfunkel and Dressler ${ }^{3}$ in which a "triptycyl moiety" is connected to three neighboring triptycyl moieties by fused benzene rings, producing an array of hexagonal pillars. The same structure has recently been suggested again by Jones. ${ }^{4}$ In this structure, graphitic strips are interconnected via $s p^{3}$ bonds at the junctions. When the widths of the constituent graphitic strips are all identical, the structure can be regarded as a honeycomb lattice with each side (line segment) of the hexagon composed of a graphitic strip perpendicular to the lattice plane. We call this a graphitic honeycomb $(\mathrm{GH})$ lattice in this paper. Although there was an integral number of fused benzene rings between two triptycyl moieties in the original suggestion, ${ }^{3}$ we study below the cases where the width of the interconnected graphitic strip is a multiple of half the number of benzene rings as well. Since the family of GH's has a hexagonal array of pores along one direction, they have potential applicability as one-dimensional ionic conductors for batteries, shape-selective catalysts, molecular sieves and absorbents, and electron emitters. We study the electronic structure and mechanical stability of the family of GH's with an $a b$ initio pseudopotential as well as the environment-dependent tight-binding method. Our calculation shows that the electronic structure has a similar size dependence to that of zigzag carbon nanotubes. It turns out that semiconducting GH's have a wider range of band gap than nanotubes, with potential applications for electronic or optical devices. The mechanical stability of GH's turns out to be comparable to that of diamond and they have fairly high bulk and shear modulus.

The remainder of the paper is organized as follows. In Sec. II, we briefly describe the method of calculation. We give a detailed description of the geometry of the $\mathrm{GH}$ and present the calculated electronic structures in Sec. III. We compare the electronic structure of the $\mathrm{GH}$ with that of the carbon nanotube. In Sec. IV, we obtain equilibrium lattice constants and elastic moduli and compare them with other carbon structures. A summary is given in the last section.

\section{COMPUTATIONAL METHOD}

We use the $a b$ initio pseudopotential method with the plane-wave basis ${ }^{5}$ for small unit cell size GH's. Since the $a b$ initio band structure is well reproduced by the environmentdependent tight-binding method, ${ }^{6}$ we use the tight-binding method with a non-orthogonal basis ${ }^{7}$ in the electronic structure calculation of larger size GH's. In the $a b$ initio calculations, we employ the local density approximation (LDA) to the density functional theory. The pseudopotential for carbon is generated through the Troullier-Martins scheme ${ }^{8}$ and cast into a fully nonlocal form. ${ }^{9}$ We adopt the Ceperly-Alder-type exchange-correlation potential ${ }^{10}$ as parametrized by Perdew and Zunger. ${ }^{11}$ The kinetic energy cutoff in the plane-wave expansion is $55 \mathrm{Ry}$. The Brillouin zone is sampled using the Monkhorst-Pack special points method ${ }^{12}$ with 8 to $14 k$ points in the irreducible part of the Brillouin zone.

\section{GEOMETRY AND THE ELECTRONIC STRUCTURE OF THE GH}

In a $\mathrm{GH}$, as shown in Fig. 1, three graphite planes meet with one another at $120^{\circ}$ forming a linear chain of $s p^{3}$ bonds at the junction. The bonds at the junction are negligibly distorted from the ideal tetrahedral bonds of diamond according to our energy minimization calculation. If the width of the side plane is $N$ times the hexagonal unit of graphite ( $N$ can be an integer or a half integer), the $\mathrm{GH}$ is designated as size $N$. In Fig. 1(a), we present a perspective view of the bulk structure of the GH of size 3. Only five hexagonal units in the pillar axis direction and seven unit cells in the perpendicular plane are shown for visual clarity. A close-up view of 


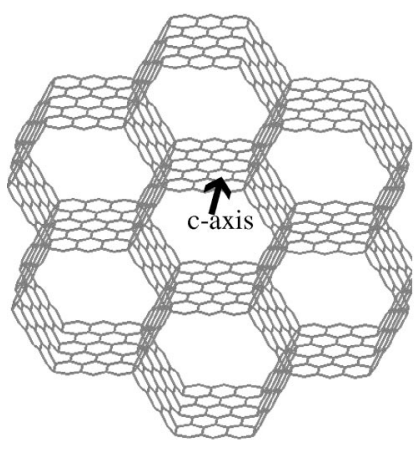

(a)

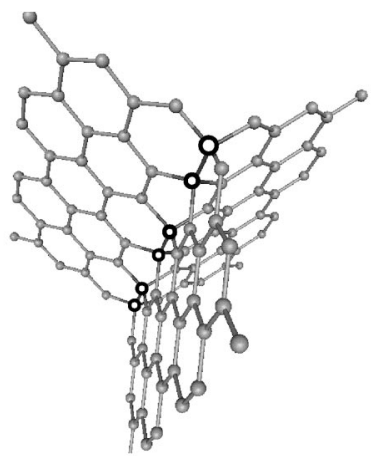

(b)

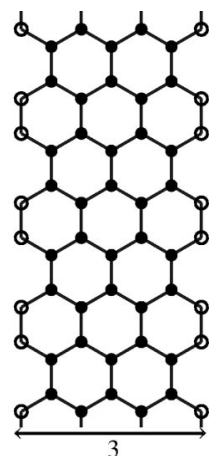

(c)
FIG. 1. Structure of the graphitic honeycomb lattice of size 3. (a) Perspective view of a portion of the lattice. Only seven unit cells of the hexagonal pillars and graphitic sheets of five hexagons high in the pillar axis ( $c$-axis) direction are presented. (b) Bonding configuration near the junction. (c) Graphitic strip of size 3. the bonding configuration near the junction is presented in Fig. 1(b). The filled circles represent the carbon atoms of $s p^{2}$ bonding in the plane and the open circles represent the carbon atoms of $s p^{3}$ bonding at the junction. Figure 1(c) shows a single graphitic side plane (the building block of the $\mathrm{GH}$ ) of size 3 .

Now we examine the electronic structure of GH's of various sizes. The results of environment-dependent tightbinding calculations on the density of states using four orbitals per carbon atom are presented in Fig. 2. The electronic structure of the GH has similar size dependence to that of zigzag nanotubes. When $N=$ (integer) $\times 3 / 2$, the GH of size $N$ is metallic, but otherwise semiconducting. We explain this size dependence as follows. Since the side planes of the GH are nearly undistorted from graphitic strips, electronic states near the Fermi level are expected to originate from $\pi$-bonded $p_{z}$ (perpendicular to the plane) orbitals as in graphite and carbon nanotubes. Let us approximate the electronic structure by the nearest-neighbor $\pi$ orbital tight-binding model. The GH of size $N$ is an assembly of strips of size $N$ as shown in Fig. 1(c). Those eigenstates of an isolated strip that have zero amplitudes on both ends are eigenstates of the $\mathrm{GH}$ as well, because states in one strip are completely decoupled from states of other strips owing to the zero amplitude at the junction. We now show that these states can all be found in the eigenstates of a zigzag carbon nanotube of an appropriate size. If we have two oppositely propagating plane waves of period $2 L$, i.e., $e^{i k_{n} x}$ and $e^{-i k_{n} x}$ (where $k_{n}=2 \pi n / 2 L$ ), a linear combination of opposite signs of these two waves results in a standing wave $[\sin (n \pi x / L)]$ which has nodes at $x=0$ and $L$. Such a standing wave is an eigenstate of the onedimensional box of width $L$. This is the situation occurring in an isolated graphite strip. Eigenstates of the nearest-neighbor $\pi$-orbital tight-binding Hamiltonian whose amplitudes are zero at both edges of the strip of size $N$ can be obtained by linear combination of two states of the $(2 N, 0)$ nanotube propagating in opposite directions around the tube. The $\pi$-orbital tight-binding bands of the $(2 N, 0)$ tube $^{13}$ can be expressed as

$$
E_{m}(k)= \pm \gamma\left[1 \pm 4 \cos \left(\frac{\sqrt{3} k a}{2}\right) \cos \left(\frac{m \pi}{2 N}\right)+4 \cos ^{2}\left(\frac{m \pi}{2 N}\right)\right]^{1 / 2},
$$

where $-\pi / \sqrt{3} \leqslant k a<\pi / \sqrt{3}$ and $m=1, \ldots, 2 N$. The wave vector $k$ here is along the tube axis and corresponds precisely to that of the GH along the $c$ axis. $\gamma$ is the nearest-neighbor

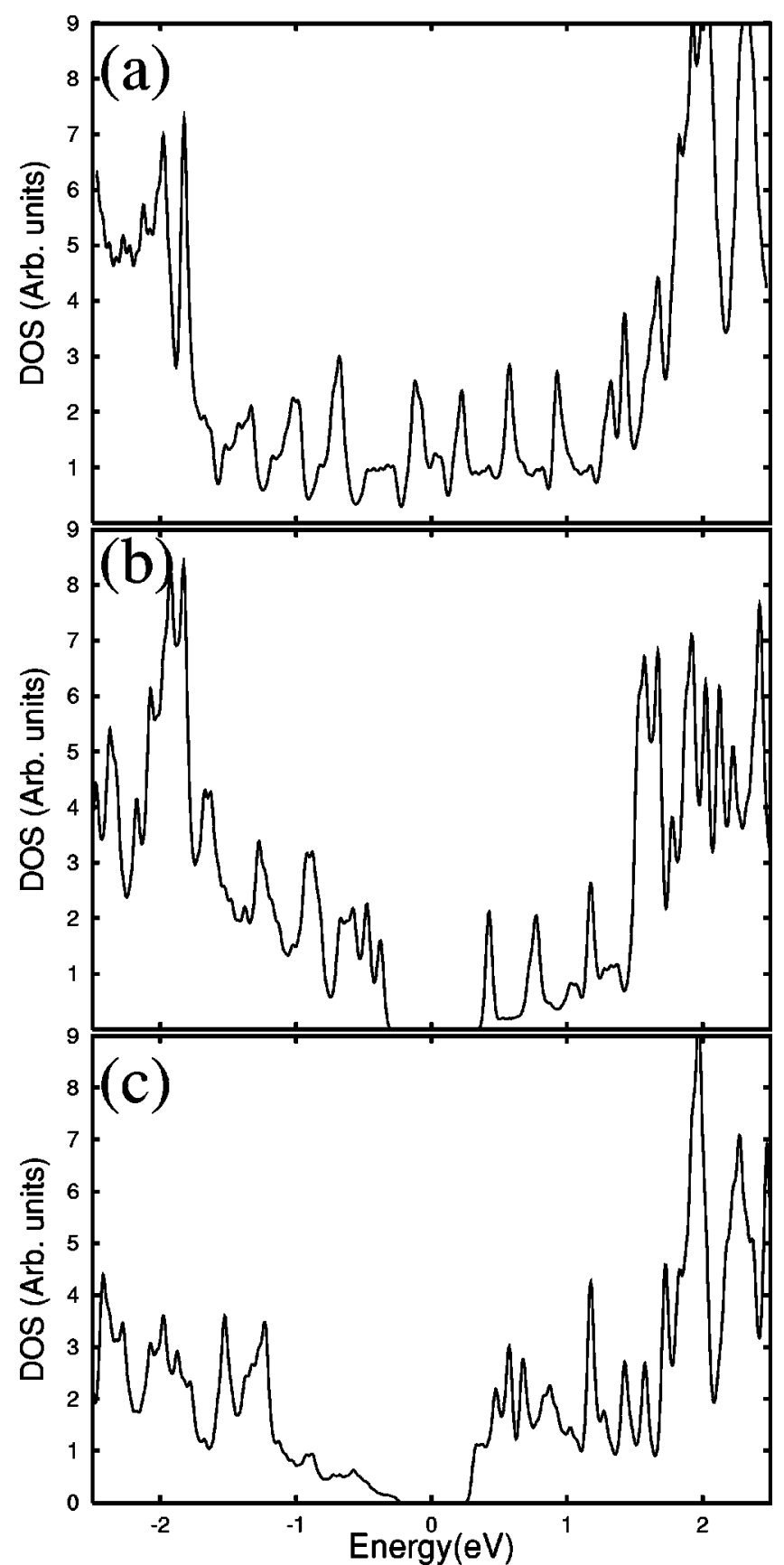

FIG. 2. Density of states of the GH of (a) size 3, (b) size 3.5, and (c) size 4 . 

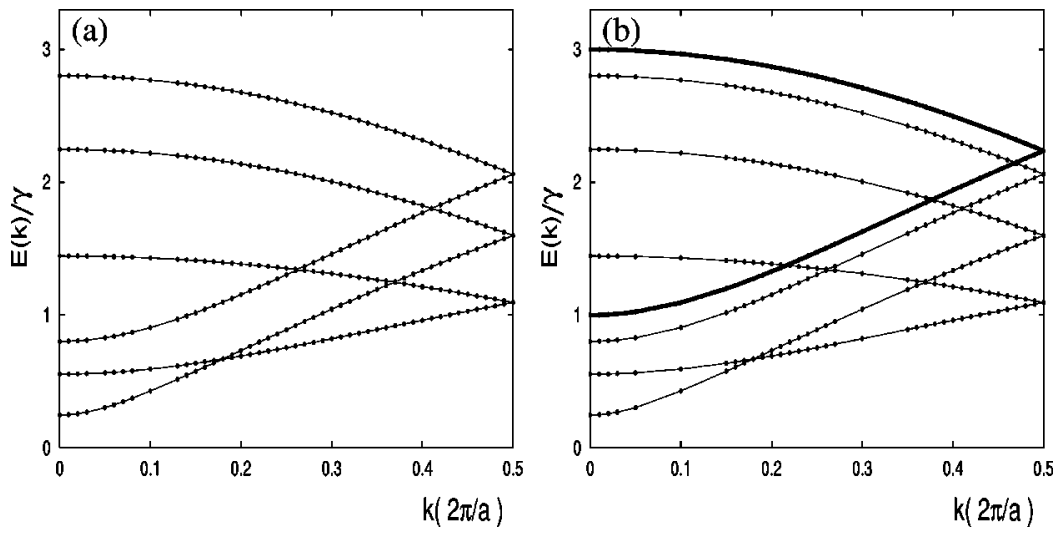

FIG. 3. The $\pi$-orbital tight-binding conduction band of (a) the graphitic strip of size 3.5 and (b) the $(7,0)$ nanotube. The heavy lines indicate the nondegenerate bands present in the nanotube only. hopping parameter $(\sim 2.7 \mathrm{eV})$. The + and - signs outside the brackets denote the conduction and valence bands, respectively. With a certain choice of the unit cell, the + and - signs inside the brackets correspond to the unfolded and folded bands. ${ }^{13}$ The folded band of $m(1 \leqslant m \leqslant 2 N-1)$ is degenerate with the unfolded band of $m^{\prime}=2 N-m$ and we have $2 N-1$ doubly degenerate bands. A linear combination of opposite signs of two degenerate states results in an eigenstate of the graphite strip. Corresponding to $m=2 N$, there exist one nondegenerate folded band and one nondegenerate unfolded band. To reiterate, we have $8 N \pi$ electrons, which generate $4 N$ conduction bands and $4 N$ valence bands in the $(2 N, 0)$ nanotube. If we count conduction bands only, we have $2 N-1$ doubly degenerate bands and two nondegenerate bands. In the graphitic strip of size $N$, we have $4 N-2$ (excluding two edge atoms) $\pi$ electrons in the unit cell. The $2 N-1$ conduction bands can be obtained by a linear combination of the corresponding degenerate states in the $(2 N, 0)$ tube. For an explicit illustration, we present the results for the graphite strip of size 3.5 and the $(7,0)$ nanotube in Fig. 3. The Fermi level is set to zero in this figure. Since we have perfect electron-hole symmetry in this model, we show only the conduction bands $(E>0)$. The bands of the graphitic strip of size 3.5 exactly coincide with the degenerate bands of the $(7,0)$ nanotube.

The above discussions focus on the $\pi$-bonded states and their dispersion in the $c$-axis direction. Now we present the band structure of the $\mathrm{GH}$ of size 2 obtained from the $a b$ initio pseudopotential calculation with the LDA in Fig. 4. Although orbitals of other than $p_{z}$ character may be involved and mixing of $\pi$ and $\sigma$ bonds is fully allowed in the ab initio calculation, the results show that the bands near the Fermi level are fairly flat along the direction perpendicular to the $c$ axis and the charge density of these states is very low (not shown) at the junction. This indicates that the above tightbinding model (giving a completely flat band in the perpendicular direction and zero charge density at the junction) is a reasonable approximation for the states near the Fermi level. The size of the gap $(\simeq 1.3 \mathrm{eV})$ also agrees with that of the tight-binding calculation. This is in contrast to other group IV intrinsic semiconductors ( $\mathrm{Si}$ and $\mathrm{Ge}$ ) where the indirect gap is badly underestimated by use of the LDA. The gap size here is significantly greater than that of carbon nanotubes. The experimentally realizable gap size of the carbon nanotubes is within $1 \mathrm{eV}$ and the typical semiconducting nanotube (with a diameter of $\sim 1.4 \mathrm{~nm}$ ) has a gap of $\sim 0.6 \mathrm{eV}$. This property may be useful for certain electronic device applications, e.g., those requiring low-leakage current. Furthermore, the gap is in practice direct at $\Gamma$ with almost no dispersion of the highest valence band and the lowest conduction band in the $\Gamma K M$ plane, with possible applications utilizing optical properties. We note that the GH of size 1 or size 0.5 (not shown) does not have any $\pi$-bonded chains in the side plane and the above argument based on graphitic strips is not applicable. The GH of size 1 turns out to be a semimetal in our ab initio calculation as demonstrated by the band structure presented in Fig. 5. The GH of size 0.5 in our convention is actually hexagonal diamond in which all atoms form $s p^{3}$ bonding. The results for the electronic structure of the previous calculation ${ }^{3}$ differ significantly from ours in that the $\mathrm{GH}$ of size 1 or size 3 has a band gap that is greater than $5 \mathrm{eV}$. The magnitude of the band gap of the size $2 \mathrm{GH}$ has also been overestimated compared with the present calculation.

While the side planes of the GH have 'armchair' edges on both sides as shown in Fig. 1(c), we can build another structure whose side planes have zigzag edges. This structure turns out to have triclinic symmetry according to our calculation. The bonding at the junction resembles the tetrahedral $\sigma$ bond of cubic diamond. Both $a b$ initio and tight-binding calculations show that, irrespective of their size, this family of structures is always metallic; they have nearly flat bands near the zone edge as in other calculations on graphitic strips with zigzag edges. ${ }^{14,15}$

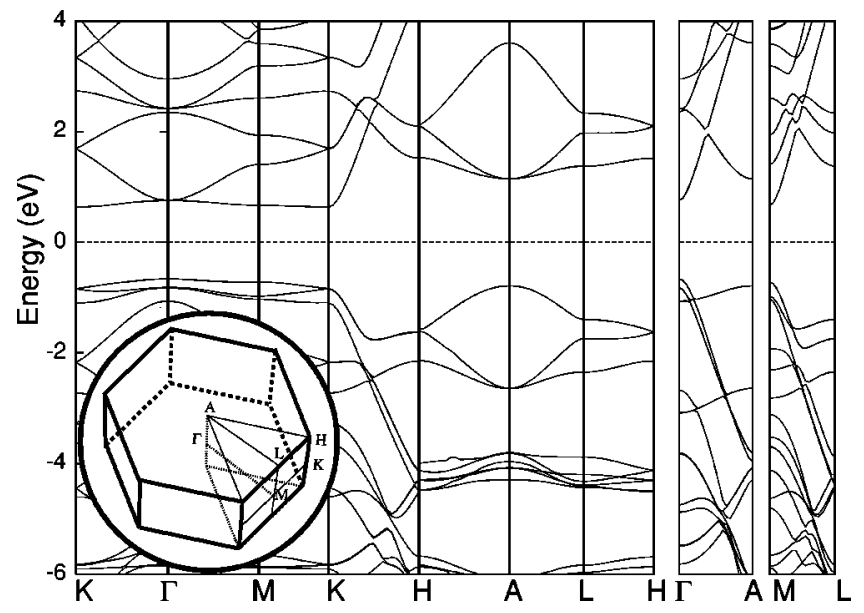

FIG. 4. Band structure of the GH of size 2 obtained using the $a b$ initio pseudopotential method with the LDA. The inset is the first Brillouin zone. 


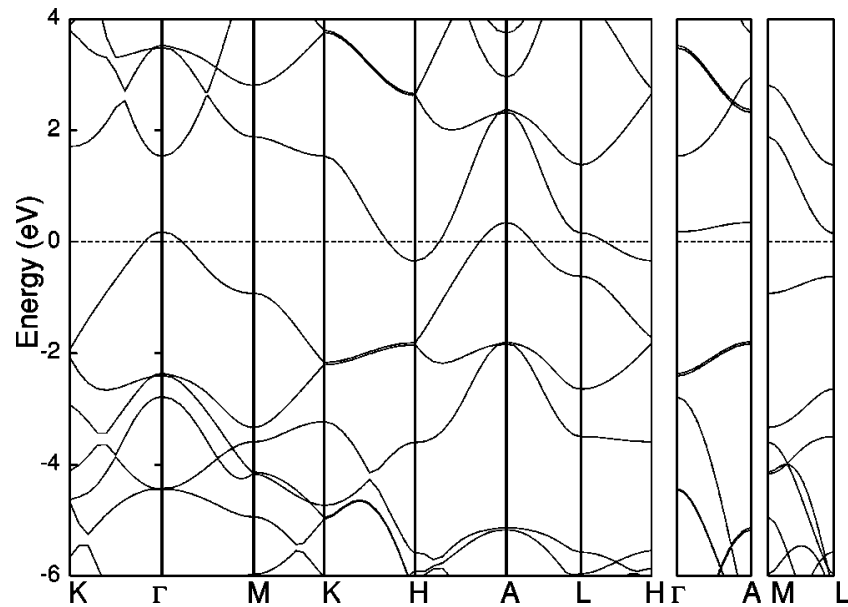

FIG. 5. Band structure of the GH of size 1 obtained using the $a b$ initio pseudopotential method with the LDA. The labeling of the first Brillouin zone is the same as in Fig. 4.

\section{STRUCTURAL STABILITY}

In this section, we investigate equilibrium lattice constants and elastic stability of GH's. Ab initio calculations for small size GH's $(1 \leqslant N \leqslant 2)$ indicate that the $s p^{2}$ bond length in the $c$-axis direction is about $1.41 \AA$ and other $s p^{2}$ bond lengths are about $1.39 \AA$, compared with the experimental graphite bond length of $1.42 \AA$, whereas the $s p^{3}$ bond length at the junction in the $c$-axis direction is about $1.53 \AA$ and other $s p^{3}$ bond lengths are about $1.52 \AA$, compared with the experimental diamond bond length of $1.545 \AA$ A. The elastic energy density of the hexagonal structure ${ }^{16}$ can be written in the form

$$
\begin{aligned}
U= & \frac{1}{2} C_{11}\left(e_{1}^{2}+e_{2}^{2}\right)+\frac{1}{2} C_{33} e_{3}^{2}+\frac{1}{2} C_{44}\left(e_{4}^{2}+e_{5}^{2}\right) \\
& +\frac{1}{2}\left(C_{11}-C_{22}\right) e_{6}^{2}+C_{12} e_{1} e_{2}+C_{13}\left(e_{1} e_{3}+e_{2} e_{3}\right),
\end{aligned}
$$

where the $e$ 's are conventional strain components. By differentiating the elastic energy density with respect to strain components, we have the following stress-strain relations:

$$
\begin{aligned}
& \sigma_{1}=C_{11} e_{1}+C_{12} e_{2}+C_{13} e_{3}, \\
& \sigma_{2}=C_{12} e_{1}+C_{11} e_{2}+C_{13} e_{3}, \\
& \sigma_{3}=C_{13} e_{1}+C_{13} e_{2}+C_{33} e_{3} .
\end{aligned}
$$

The Born criteria ${ }^{17}$ for the stability of the hexagonal crystal require that $C_{11}, C_{11}-C_{12}, C_{44}$, and $\left(C_{11}+C_{12}\right) C_{13}$ $-2 C_{13}^{2}$ should all be greater than zero. As shown in Table I, the GH's well satisfy the criteria. From these elastic constants, we can calculate Poisson's ratio, Young's modulus, and bulk modulus. If we load a tensile stress on the GH specimen in the $c$-axis direction with the sides of the specimen left free (i.e., $\sigma_{1}=0$ and $\sigma_{2}=0$ ), we have the Young's modulus
TABLE I. Elastic moduli of GH's in GPa.

\begin{tabular}{lccccccc}
\hline \hline & $C_{11}$ & $C_{12}$ & $C_{13}$ & $C_{33}$ & $C_{44}$ & $Y_{11}$ & $Y_{33}$ \\
\hline Size 1/2 & 1254 & 103 & 13 & 1338 & 485 & 1338 & 1235 \\
Size 1 & 529 & 118 & 0 & 1132 & 279 & 1132 & 500 \\
Size 3/2 & 382 & 118 & 24 & 765 & 174 & 763 & 345 \\
Size 2 & 324 & 15 & 0 & 618 & 132 & 618 & 324 \\
\hline \hline
\end{tabular}

$$
Y_{33}=\frac{\sigma_{3}}{e_{3}}=C_{33}+C_{13}\left(\frac{e_{1}}{e_{3}}+\frac{e_{2}}{e_{3}}\right) \text {, }
$$

where the Poisson's ratios are

$$
\frac{e_{1}}{e_{3}}=\frac{e_{2}}{e_{3}}=-\frac{C_{11} C_{13}-C_{12} C_{13}}{C_{11}^{2}-C_{12}^{2}} .
$$

In the same way, the Young's modulus in the direction perpendicular to the $c$ axis is

$$
Y_{11}=C_{11}+C_{12} \frac{e_{2}}{e_{1}}+C_{13} \frac{e_{3}}{e_{1}},
$$

where

$$
\begin{gathered}
\frac{e_{2}}{e_{1}}=-\frac{C_{33} C_{12}-C_{13}^{2}}{C_{11} C_{33}-C_{13}^{2}}, \\
\frac{e_{3}}{e_{1}}=-\frac{C_{11} C_{13}-C_{13} C_{12}}{C_{11} C_{33}-C_{13}^{2}} .
\end{gathered}
$$

We also investigate the cohesive energy and bulk modulus of GH's and compare them with other carbon structures. The cohesive energies are presented in Table II. The bulk modulus $^{18}$ of the hexagonal crystal is obtained from the relation

$$
B=\frac{\Delta C_{33}+2 C_{13}}{\Delta+2},
$$

where

$$
\Delta=\frac{C_{11}+C_{12}-2 C_{13}}{C_{33}-C_{13}},
$$

TABLE II. Cohesive energy of GH's, diamond, and graphite in eV/atom.

\begin{tabular}{lcc}
\hline \hline & Present calc. & Other works \\
\hline Cubic diamond & 8.754 & $7.2^{\mathrm{a}}, 8.17^{\mathrm{b}}$ \\
Graphite & 8.846 & $7.4^{\mathrm{a}}$ \\
Size $1 / 2$ (hex. diamond) & 8.749 & $(\text { c.d. }-0.002)^{\mathrm{c}}, 8.14^{\mathrm{b}}$ \\
Size 1 & 8.313 & \\
Size 3/2 & 8.563 & \\
Size 2 & 8.641 & \\
\hline \hline
\end{tabular}

${ }^{\mathrm{a}}$ Reference 20.

${ }^{\mathrm{b}}$ Reference 19.

${ }^{\mathrm{c}}$ Reference 1 . The number is with respect to cubic diamond (c.d.). 
TABLE III. Bulk modulus of GH's and diamond in GPa.

\begin{tabular}{lcc}
\hline \hline$B$ & Present calc. & Other work \\
\hline Cubic diamond & $458^{\mathrm{a}}$ & $449^{\mathrm{b}}, 457^{\mathrm{b}}, 444^{\mathrm{c}}$ \\
Size $1 / 2$ (hex. diamond) & 456 & $448^{\mathrm{b}}, 440^{\mathrm{c}}$ \\
Size 1 & 252 & \\
Size $3 / 2$ & 214 & \\
Size 2 & 133 & \\
\hline \hline
\end{tabular}

${ }^{a}$ For a cubic crystal, $B=\left(C_{11}+2 C_{12}\right) / 3$.

${ }^{b}$ Reference 1 .

${ }^{\mathrm{c}}$ Reference 19 .

and presented in Table III. The difference in the heat of formation between diamond and graphite in the previous calculation $^{3}$ is roughly the same as the corresponding difference in the cohesive energy in our calculation. But the previous calculation with the semiempirical method seems to be inaccurate in that the GH's of size 2 and size 3 are more stable than diamond, which is quite unlikely from the experimental point of view. In our calculation, the cohesive energy of the $\mathrm{GH}$ of size 2 is $0.1 \mathrm{eV} /$ atom smaller than that of diamond. This means that the $\mathrm{GH}$ is energetically more stable than the BC-8 structure, which is metastable at atmospheric pressure and room temperature with a reported cohe- sive energy of $0.7 \mathrm{eV} /$ atom smaller than that of diamond. ${ }^{19,1}$ The bulk modulus is fairly large as shown in Table III. For comparison, the bulk modulus of silicon is $99 \mathrm{GPa}$. Since the cohesive energy is comparable to diamond and the Born criteria are satisfied as mentioned above, GH's are expected to be stable once they are formed.

\section{SUMMARY}

We have calculated the electronic structure and mechanical properties of the GH's using an ab initio pseudopotential as well as the environment-dependent tight-binding method. The electronic structure of the GH has a similar size dependence to that of zigzag nanotubes. When the size $N$ (defined in Sec. III) is equal to an (integer) times $3 / 2$, the $\mathrm{GH}$ is metallic, and otherwise semiconducting. The $\mathrm{GH}$ is elastically stable and has fairly high shear modulus. Having a similar electronic structure to the zigzag nanotube and a higher shear modulus in the plane perpendicular to the $c$ axis (and a greater band gap in some cases) than nanotube ropes, the GH may have interesting applications beyond those of nanotubes.

\section{ACKNOWLEDGMENTS}

This work was supported by the BK21 Project of KRF and the Ministry of Science and Technology.
${ }^{1}$ C. Mailhiot and A. K. McMahan, Phys. Rev. B 44, 11578 (1991).

${ }^{2}$ A. T. Balaban, D. J. Klein, and C. A. Folden, Chem. Phys. Lett. 14, 266 (1994)

${ }^{3}$ H. R. Karfunkel and T. Dressler, J. Am. Chem. Soc. 114, 2285 (1992).

${ }^{4}$ D. Jones, Nature (London) 394, 133 (1998).

${ }^{5}$ J. Ihm, A. Zunger, and M. L. Cohen, J. Phys. C 12, 4409 (1979).

${ }^{6}$ M. S. Tang, C. Z. Wang, C. T. Chan, and K. M. Ho, Phys. Rev. B 53, 979 (1996).

${ }^{7}$ M. J. Mehl and D. A. Papaconstantopoulos, Phys. Rev. B 54, 4519 (1996).

${ }^{8}$ N. Troullier and J. L. Martins, Phys. Rev. B 43, 1993 (1991).

${ }^{9}$ L. Kleinman and D. M. Bylander, Phys. Rev. Lett. 48, 1425 (1982).

${ }^{10}$ D. M. Ceperley and B. J. Alder, Phys. Rev. Lett. 45, 566 (1980).

${ }^{11}$ J. P. Perdew and A. Zunger, Phys. Rev. B 23, 5048 (1981).
${ }^{12}$ H. J. Monkhorst and J. D. Pack, Phys. Rev. B 13, 5188 (1976).

${ }^{13}$ R. Saito, M. Fujita, G. Dresselhaus, and M. S. Dresselhaus, Phys. Rev. B 46, 1804 (1992).

${ }^{14}$ Y. Miyamoto, K. Nakada, and M. Fujita, Phys. Rev. B 59, 9858 (1999).

${ }^{15}$ M. Fujita, K. Wakabayashi, K. Nakada, and K. Kusakabe, J. Phys. Soc. Jpn. 65, 1920 (1996).

${ }^{16}$ N. J. Park, H. Klein, and E. Dahelm-Klein, Physical Properties of Textured Materials (Cuvillier, Gottingen, 1993).

${ }^{17}$ A. Y. Liu, Phys. Rev. B 50, 10362 (1994).

${ }^{18}$ P. Y. Yu and M. Cardona, Fundamentals of Semiconductors (Springer, Berlin, 1995).

${ }^{19}$ S. Fahy and S. G. Louie, Phys. Rev. B 36, 3373 (1987).

${ }^{20}$ M. S. Dresselhaus, G. Dresselhaus, and P. C. Eklund, Science of Fullerenes and Carbon Nanotubes (Academic, San Diego, 1996). 